Kragujevac Journal of Mathematics

Volume 45(1) (2021), Pages 47-61.

\title{
$\rho$-ATTRACTIVE ELEMENTS IN MODULAR FUNCTION SPACES
}

\author{
H. IQBAL ${ }^{1}$, M. ABBAS ${ }^{2}$, AND S. H. KHAN ${ }^{3}$
}

\begin{abstract}
In this paper, we introduce the notion of $\rho$-attractive elements in modular function spaces. A new class of mappings called $\rho$ - $k$-nonspreading mappings is also introduced. Making a good use of the two notions, we first prove existence results and then some approximation results in the setup of modular function spaces. An example is presented to support the results proved herein.
\end{abstract}

\section{INTRODUCTION AND PRELIMINARIES}

The notion of attractive points of nonlinear mappings in Hilbert spaces was coined by Takahashi and Takeuchi [16] in 2011.

Let $E$ be a nonempty subset of a Hilbert space $H$ and $T: E \rightarrow H$ then the set of attractive points $A(T)$ is given by,

$$
A(T)=\{z \in H:\|T x-z\| \leq\|x-z\| \text { for all } x \in E\} .
$$

They proved an existence result on attractive points for the so-called hybrid mappings in a Hilbert space. They went on to prove a weak convergence theorem of Mann-type without closedness.

Motivated by the idea of Takahashi et al. [17], study of attractive points gained momentum. Several different classes of mappings were introduced. Kohsaka et al. [8] presented a new class of mappings called nonspreading mappings.

A mapping $T: E \rightarrow E$ is said to be nonspreading mapping if for any $x, y \in E$,

$$
2\|T x-T y\|^{2} \leq\|x-T y\|^{2}+\|T x-y\|^{2} .
$$

Suantai et al. [15], using Hausdorff metric, introduced the class of generalized nonspreading mappings, known as $k$-nonspreading multivalued mappings. Kaewkhao et

Key words and phrases. Attractive points, modular spaces, nonspreading mappings, modular functions.

2010 Mathematics Subject Classification. Primary: 47H10. Secondary: 47H09, 47J25.

DOI 10.46793/KgJMat2101.047I

Received: March 29, 2018.

Accepted: September 03, 2018. 
al. [1] studied the attractive points and convergence theorems for normally generalized hybrid mappings in $C A T(0)$ spaces in 2015.

In the same year, Zheng [18] proved strong and weak convergence theorem of the Ishikawa iteration for an $(\alpha, \beta)$-generalized hybrid mapping in a uniformly convex Banach space. Kunwai et al. [5] proved an attractive point theorem for normally generalized hybrid mappings in $C A T(0)$ spaces under certain conditions. Recently, fixed point theory in modular function spaces has gained interest of many mathematicians. The idea of modular function spaces was established by Nakano in [13] and was improved and generalized by Musielak and Orlicz [12]. Later on, Khamsi et al. [11] introduced the fixed point theory in modular function spaces and proved Banach contraction principle in modular function spaces (also see [6]). Kuaket and Kumam [10] established some fixed point for generalized contraction mappings in modular function spaces. Dehaish and Kozolwoski [2], proved results on approximating fixed points in modular function spaces for the first time. Recently, Khan et al. [14] successfully handled the problem of approximating fixed points for multivalued $\rho$-quasi nonexpansive mappings in modular function spaces. Ilchev and Zlatanov [3] presented some sufficient conditions for the existence and uniqueness of best proximity points and fixed points for cyclic Kannan maps in modular function spaces. For further discussion in modular spaces see $[4,9,19]$.

The above efforts stimulate us to define attractive elements in the setting of modular function spaces. Another purpose of this paper is to define a class of $\rho-k$-nonspreading mappings. This will lead us proving existence and approximation results for attractive elements in modular function spaces. Towards the end of this paper, our results will be vindicated using some examples.

Let us recall some basic definitions and notions which can be found in [7]. Let $\Omega$ be a nonempty set and $\Sigma$ be a nontrivial $\sigma$-algebra of subsets of $\Omega$. Let $P$ be a nontrivial $\delta$-ring of subsets of $\Omega$ which means that $P$ is closed with respect to forming of countable intersections, and finite unions and differences. Assume further that $E \cap A \in P$ for any $E \in P$ and $A \in \sum$. Let us assume that there exists an increasing sequence of sets $K_{n} \in P$ such that $\Omega=\bigcup K_{n}$. By $\mathscr{E}$ we denote the linear space of all simple functions with supports from $P . \mathcal{M}_{\infty}$ represents the space of all extended measurable functions, that is, all functions $f: \Omega \rightarrow[-\infty, \infty]$ such that there exists a sequence $\left\{g_{n}\right\} \subset \mathscr{E},\left|g_{n}\right| \leq|f|$ and $g_{n}(\omega) \rightarrow f(\omega)$ for all $\omega \in \Omega$.

Definition 1.1. Let $\rho: \mathcal{M}_{\infty} \rightarrow[0, \infty]$ be a nontrivial, convex, and even function. We say that $\rho$ is a regular convex function pseudomodular if

(a) $\rho(0)=0$;

(b) $\rho$ is monotone, i.e., $|f(\omega)| \leq|g(\omega)|$ for any $\omega \in \Omega$ implies $\rho(f) \leq \rho(g)$, where $f, g \in \mathcal{M}_{\infty}$

(c) $\rho$ is orthogonally subadditive, i.e., $\rho\left(f 1_{A \cup B}\right) \leq \rho\left(f 1_{A}\right)+\rho\left(f 1_{B}\right)$ for any $A, B \in \Sigma$ such that $A \cup B \neq \phi, f \in \mathcal{M}_{\infty}$; 
(d) $\rho$ has Fatou property, that is, $\left|f_{n}(\omega)\right| \uparrow|f(\omega)|$ for all $\omega \in \Omega$ implies $\rho\left(f_{n}\right) \uparrow \rho(f)$, where $f \in \mathcal{M}_{\infty}$;

(e) $\rho$ is order continuous in $\mathscr{E}$, i.e., $g_{n} \in \mathscr{E}$, and $\left|g_{n}(\omega)\right| \downarrow 0$ implies $\rho\left(g_{n}\right) \downarrow 0$.

We say that a set $A \in \Sigma$ is $\rho$-null if $\rho\left(g 1_{A}\right)=0$ for every $g \in \mathscr{E}$. A property holds $\rho$-almost everywhere ( $\rho$-a.e.) if the set $\{\omega \in \Omega: p(\omega)$ does not hold $\}$ is $\rho$-null. We identify any pair of measurable sets whose symmetric difference is $\rho$-null as well as any pair of measurable functions differing only on a $\rho$-null set. With this in mind we define $\mathcal{M}=\left\{f \in \mathcal{M}_{\infty}:|f(\omega)|<\infty \rho\right.$-a.e. $\}$ where each $f \in \mathcal{M}$ is actually an equivalence class of functions equal $\rho$-a.e. rather than an individual function.

Definition 1.2. Let $\rho$ be a regular convex function pseudomodular. Then, we say that $\rho$ is a regular convex function modular if $\rho(f)=0$ implies that $f=0 \rho$-a.e.

The class of all nonzero regular convex function modular defined on $\Omega$ is denoted by $\Re$.

Definition 1.3. The convex function modular $\rho$ defines the modular function space $\mathbb{L}_{\rho}$ as

$$
\mathbb{L}_{\rho}=\left\{f \in \mathcal{M}_{\infty}: \rho(\lambda f) \rightarrow 0 \text { as } \lambda \rightarrow 0\right\} .
$$

Generally, the modular $\rho$ is not subadditive and hence doesn't behave like a norm. However, the modular space $\mathbb{L}_{\rho}$ can be equipped with an $F$-norm defined by

$$
\|f\|_{\rho}=\inf \left\{\alpha>0: \rho\left(\frac{f}{\alpha}\right) \leq \alpha\right\} .
$$

If $\rho$ is a convex modular,

$$
\|f\|_{\rho}=\inf \left\{\alpha>0: \rho\left(\frac{f}{\alpha}\right) \leq 1\right\}
$$

defines a norm on the modular space $\mathbb{L}_{\rho}$, and is called the Luxemburg norm. The following definitions will be needed in this paper.

Definition 1.4. Let $\mathbb{L}_{\rho}$ be a modular space. Then

(a) the sequence $\left\{f_{n}\right\} \subset \mathbb{L}_{\rho}$ is said to be $\rho$-convergent to $f \in \mathbb{L}_{\rho}$ if $\rho\left(f_{n}-f\right) \rightarrow 0$ as $n \rightarrow \infty$

(b) the sequence $\left\{f_{n}\right\} \subset \mathbb{L}_{\rho}$ is said to be $\rho$-Cauchy if $\rho\left(f_{n}-f_{m}\right) \rightarrow 0$ as $n$ and $m$ approach $\infty$;

(c) we say that $\mathbb{L}_{\rho}$ is $\rho$-complete if and only if any $\rho$-Cauchy sequence in $\mathbb{L}_{\rho}$ is $\rho$-convergent.

Definition 1.5. A subset $E$ of $\mathbb{L}_{\rho}$ is called

(a) $\rho$-closed if the $\rho$-limit of a $\rho$-convergent sequence of $E$ always belongs to $E$;

(b) $\rho$-compact if every sequence in $E$ has a $\rho$-convergent subsequence in $E$;

(c) $\rho$-bounded if $\delta_{\rho}(E)=\sup \{\rho(f-g): f, g \in E\}<\infty$; 
(d) the $\rho$-distance between $f$ and $E$ is defined as:

$$
d_{\rho}(f, E)=\inf \{\rho(f-j): j \in E\} .
$$

The terminology defined for $\rho$ is similar to metric spaces but $\rho$ does not satisfy triangle inequality. Hence, if a sequence in $\mathbb{L}_{\rho}$ is $\rho$-convergent it does not imply $\rho$-Cauchy. This is only true if and only if $\rho$ satisfies $\Delta_{2}$-condition.

Definition 1.6. The modular function $\rho$ is said to satisfy the $\Delta_{2}$-condition if $\rho\left(2 f_{n}\right) \rightarrow 0$ as $n$ approaches $\infty$, whenever $\rho\left(f_{n}\right) \rightarrow 0$ as $n$ approaches $\infty$.

The modular $\rho$ satisfies some uniform convexity type properties. A few of those are given below which can be found in [7].

Definition 1.7. Let $\rho \in \Re$.

(a) Let $r>0, \epsilon>0$. Define,

$$
D_{1}(r, \epsilon)=\left\{(f, h): f, h \in \mathbb{L}_{p}, \rho(f) \leq r, \rho(h) \leq r, \rho(f-h) \geq \epsilon r\right\} .
$$

Let

$$
\delta_{1}(r, \epsilon)=\inf \left\{1-\frac{1}{r} \rho\left(\frac{f+h}{2}\right):(f, h) \in D_{1}(r, \epsilon)\right\}, \quad \text { if } D_{1}(r, \epsilon) \neq \phi,
$$

and $\delta_{1}(r, \epsilon)=1$ if $D_{1}(r, \epsilon)=\phi$. We say that $\rho$ satisfies $(U C 1)$ if for every $r>0$, $\epsilon>0, \delta_{1}(r, \epsilon)>0$. Note that for every $r>0, D_{1}(r, \epsilon) \neq \phi$ for every $\epsilon>0$ small enough.

(b) We say that $\rho$ satisfies (UUC1) if for every $s \geq 0, \epsilon>0$, there exists $\eta_{1}(s, \epsilon)>0$ depending only upon $s$ and $\epsilon$ such that $\delta_{1}(r, \epsilon)>\eta_{1}(s, \epsilon)>0$ for any $r>s$.

(c) We say that $\rho$ satisfies $(U U C 2)$ if for every $s \geq 0, \epsilon>0$, there exists $\eta_{2}(s, \epsilon)>0$ depending upon $s$ and $\epsilon$ such that $\delta_{2}(r, \epsilon)>\eta_{2}(s, \epsilon)>0$ for any $r>s$.

Note that $(U C 1)$ implies $(U U C 1)$ and $(U C C 1)$ implies (UUC2). If $\rho \in \Re$ satisfies $\Delta_{2}$, then $(U U C 2)$ and $(U C C 1)$ are equivalent (see [9]).

Definition 1.8. We will say that $\rho$ is uniformly continuous if for every $\epsilon>0$ and $R>0$, there exists $\delta>0$ such that

$$
|\rho(g)-\rho(g+h)|<\epsilon \quad \text { if } \quad \rho(h) \leq \delta, \rho(g) \leq R .
$$

A sequence $\left\{t_{n}\right\} \subset(0,1)$ is called bounded away from 0 if there exists $a>0$ such that $t_{n} \geq a$ for every $n \in \mathbb{N}$. Similarly, $\left\{t_{n}\right\} \subset(0,1)$ is called bounded away from 1 if there exists $b<1$ such that $t_{n} \leq b$ for every $n \in \mathbb{N}$. The following lemma helpful in studying the convergence of fixed points as well as attractive elements in the (UUC1) modular function spaces.

Lemma 1.1. Let $\rho \in \Re$ satisfy $(U U C 1)$ and let $\left\{t_{n}\right\} \subset(0,1)$ be bounded away from 0 and 1 . If there exists $R \geq 0$ such that

$$
\limsup _{n \rightarrow \infty} \rho\left(f_{n}\right) \leq R, \quad \limsup _{n \rightarrow \infty} \rho\left(g_{n}\right) \leq R \quad \text { and } \quad \lim _{n \rightarrow \infty} \rho\left(t_{n} f_{n}+\left(1-t_{n}\right) g_{n}\right)=R,
$$

then $\lim _{n \rightarrow \infty} \rho\left(f_{n}-g_{n}\right)=0$. 
Since the modular function space doesn't satisfy the triangle inequality so, the following theorem is useful.

Theorem 1.1. Let $\rho \in \Re$ satisfy $\Delta_{2}$-condition. Let $\left\{f_{n}\right\}$ and $\left\{g_{n}\right\}$ be two sequences in $\mathbb{L}_{\rho}$. Then,

$$
\lim _{n \rightarrow \infty} \rho\left(g_{n}\right)=0 \quad \text { implies } \quad \limsup _{n \rightarrow \infty} \rho\left(f_{n}+g_{n}\right)=\lim _{n \rightarrow \infty} \rho\left(f_{n}\right)
$$

and

$$
\lim _{n \rightarrow \infty} \rho\left(g_{n}\right)=0 \quad \text { implies } \quad \liminf _{n \rightarrow \infty} \rho\left(f_{n}+g_{n}\right)=\lim _{n \rightarrow \infty} \rho\left(f_{n}\right) .
$$

The notion of a $\rho$-type is a powerful tool that will be used in our result.

Definition 1.9. Let $E \subset \mathbb{L}_{p}$ be convex and $\rho$-bounded. A function $\tau: E \rightarrow[0, \infty]$ is called a $\rho$-type (or shortly a type) if there exists a sequence $\left\{g_{k}\right\}$ of elements of $E$ such that for any $f \in E$ there holds $\tau(f)=\limsup _{k \rightarrow \infty} \rho\left(g_{k}-f\right)$.

The following lemma [7] establishes an important minimizing sequence property of uniformly convex modular function spaces which is used proving existence of fixed points.

Lemma 1.2. Assume that $\rho \in \Re$ is (UUC1). Let $E$ be a $\rho$-closed $\rho$-bounded convex nonempty subset of $\mathbb{L}_{\rho}$. Let $\tau$ be a $\rho$-type defined on $E$. Then, any minimizing sequence of $\tau$ is $\rho$-convergent. Its $\rho$-limit is independent of the minimizing sequence.

Since our goal is to prove existence of attractive elements without the condition of $\rho$-closedness. The following is the modified version of the above lemma which can be proved exactly on lines of [7].

Lemma 1.3. Assume that $\rho \in \Re$ is (UUC1). Let $E$ be a $\rho$-bounded convex nonempty subset of $\mathbb{L}_{\rho}$. Let $\tau$ be a $\rho$-type defined on $E$. Then, any minimizing sequence of $\tau$ is $\rho$-convergent in $\mathbb{L}_{\rho}$. Its $\rho$-limit is independent of the minimizing sequence.

Definition 1.10. Let $\rho \in \Re$. The growth function $\omega_{\rho}$ of a function modular $\rho$ is defined as:

$$
\omega_{\rho}(\beta)=\sup \left\{\frac{\rho(\beta f)}{\rho(f)}, 0 \leq \rho(f)<\infty\right\}, \quad \text { for all } 0 \leq \beta<\infty .
$$

Notice that whenever $\beta \in[0,1], \omega_{\rho}(\beta) \leq 1$.

Let $T: E \rightarrow E$ be a mapping then a point $x \in E$ is said to be a fixed point of $T$ if $x=T x$. We denote the set of fixed points by $F(T)$. A mapping $T$ is said to be

(a) $\rho$-nonexpansive if $\rho(T f-T g) \leq \rho(f-g)$ for all $f, g \in E$;

(b) $\rho$-quasi-nonexpansive mapping if $\rho(T f-g) \leq \rho(f-g)$ for all $f \in E$ and $g \in F(T)$. 


\section{MAin Results}

In this section, we introduce a new class of $\rho-k$-nonspreading mappings and present the concept $\rho$-attractive elements. Then, we prove an existence and some convergence results.

Definition 2.1. Let $\rho \in \Re$. Let $T: E \rightarrow \mathbb{L}_{\rho}$ then $T$ is a $\rho-k$-nonspreading mapping if there exists a $k>0$ such that

$$
\rho^{2}(T f-T g) \leq k\left(\rho^{2}(f-T g)+\rho^{2}(T f-g)\right),
$$

for all $f, g \in E$.

A $\rho-\frac{1}{2}$-nonspreading mapping with $F(T) \neq \phi$ is $\rho$-quasi nonexpansive. In fact, if $g$ is a fixed point of $T$, then in Definition 2.1, with $k=\frac{1}{2}$, we have

$$
2 \rho^{2}(g-T f) \leq \rho^{2}(g-f)+\rho^{2}(g-T f),
$$

and, hence

$$
\rho^{2}(T f-g) \leq \rho^{2}(f-g)
$$

This implies,

$$
\rho(T f-g) \leq \rho(f-g) .
$$

Now, we give an example of a $\rho-k$-nonspreading mapping which is not a $\rho$ nonexpansive mapping.

Example 2.1. Let the real number system $\mathbb{R}$ be the space modulared as $\rho(f)=|f|^{k}$ for $k \geq 1$. Let $E=\left\{f \in \mathbb{L}_{\rho}:-3<f<2\right\}$ and

$$
T f= \begin{cases}\frac{|f|-1}{2}, & -2<f<2, \\ \frac{-|f|}{|f|+1}, & -3<f \leq-2 .\end{cases}
$$

It is easy to see that $T$ is a $\rho-\frac{1}{2}$-nonspreading mapping. However, $T$ is not a $\rho$-nonexpansive mapping since if $f=-2$ and $g=-1.5$, then

$$
\rho(T f-T g)=\left|\frac{-2}{3}-\frac{1}{4}\right|^{k}=\left|\frac{11}{12}\right|^{k}>\rho(f-g)=|-2+1.5|^{k}=\left|\frac{1}{2}\right|^{k} .
$$

Definition 2.2. Let $\rho$ be a convex function modular. Let $E$ be a nonempty subset of $\mathbb{L}_{\rho}$ and $T: E \rightarrow E$ be a mapping then a function $g \in \mathbb{L}_{\rho}$ is called a $\rho$-attractive element of $T$ if for all $f \in E$, we have $\rho(T f-g) \leq \rho(f-g)$. Let $A_{\rho}(T)$ denote the set of $\rho$-attractive elements, i.e., $A_{\rho}(T)=\left\{g \in \mathbb{L}_{\rho}: \rho(T f-g) \leq \rho(f-g)\right.$ for all $\left.f \in E\right\}$.

First of all, we will give some useful properties of $A_{\rho}(T)$.

Lemma 2.1. Let $\rho \in \Re$ and be uniformly continuous. Let $E$ be a nonempty subset of $\mathbb{L}_{\rho}$ and $T: E \rightarrow \mathbb{L}_{\rho}$, with $A_{\rho}(T) \neq \phi$. Then $A_{\rho}(T)$ is closed. 
Proof. Let $\left\{g_{n}\right\} \subset A_{\rho}(T)$ such that $\lim _{n \rightarrow \infty} \rho\left(g_{n}-g\right)=0$. Then for any $f \in E$, we have

$$
\rho(T f-g)=\rho\left(\left(T f-g_{n}\right)-\left(g-g_{n}\right)\right) .
$$

Then taking limit as $n \rightarrow \infty$ in (2.1) and using the uniform continuity of $\rho$, we get

$$
\rho(T f-g) \leq \lim _{n \rightarrow \infty} \rho\left(T f-g_{n}\right) \leq \lim _{n \rightarrow \infty} \rho\left(f-g_{n}\right)=\rho(f-g) .
$$

This shows $g \in A_{\rho}(T)$. Hence, $A_{\rho}(T)$ is closed.

An attractive point need not be a fixed point. However, if a mapping $T: E \rightarrow E$ is $\rho$-quasi nonexpansive then the $\rho$-attractive elements lying in $E$ are also its fixed points.

Lemma 2.2. Let $\rho \in \Re$. Let $E$ be a nonempty subset of $\mathbb{L}_{\rho}$ and $T: E \rightarrow \mathbb{L}_{\rho}$ be a $\rho$-quasi nonexpansive mapping. Then $A_{\rho}(T) \cap E=F(T)$.

Proof. Let $g \in A_{\rho}(T) \cap E$, then $\rho(T f-g) \leq \rho(f-g)$ for all $f \in E$. In particular, let $f=g \in E$, then we have $\rho(T f-f) \leq \rho(f-f)=\rho(0)=0$. Hence, $T f=f$ showing $f \in F(T)$. Conversely, since $T$ is $\rho$-quasi nonexpansive, then for any $h \in F(T)$ and $f \in E$, we get $\rho(T f-h) \leq \rho(f-h)$. Then, clearly $h \in A_{\rho}(T)$.

Now, we will prove existence of a $\rho$-attractive point for $\rho-k$-nonspreading mapping for $k \in\left(0, \frac{1}{2}\right]$.

Theorem 2.1. Assume that $\mathbb{L}_{\rho}$ is complete, $\rho \in \Re$ is (UUC1) and uniformly continuous. Let $E$ be a nonempty $\rho$-bounded convex subset of $\mathbb{L}_{\rho}$. Let $T: E \rightarrow E$ be a $\rho-k$-nonspreading mapping with $k \in\left(0, \frac{1}{2}\right]$. Then $T$ has a $\rho$-attractive point.

Proof. Let $\left\{f_{0}\right\} \in E$. Define the $\rho$-type, $\tau: E \rightarrow[0, \infty]$ by

$$
\tau(f)=\limsup _{n \rightarrow \infty} \rho\left(f-T^{n}\left(f_{0}\right)\right) .
$$

Then by Lemma 1.3, there exists a minimizing sequence, say, $\left\{g_{n}\right\}$, of $\tau$ such that $\tau\left(g_{n}\right)=\inf _{f \in E} \tau(f)$. Since $\left\{T^{n}\left(f_{0}\right)\right\} \subset E$ and $E$ is $\rho$-bounded we have

$$
\tau(f) \leq \delta_{\rho}(E)<\infty, \quad \text { for every } f \in E,
$$

and

$$
\tau(T f)=\limsup _{n \rightarrow \infty} \rho\left(T f-T^{n}\left(f_{0}\right)\right)
$$

Now,

$$
\rho^{2}\left(T^{n}\left(f_{0}\right)-T f\right) \leq k\left(\rho^{2}\left(T f-T^{n-1}\left(f_{0}\right)\right)+\rho^{2}\left(f-T^{n}\left(f_{0}\right)\right)\right) .
$$

Taking $n \rightarrow \infty$ implies,

$$
\limsup _{n \rightarrow \infty} \rho^{2}\left(T^{n}\left(f_{0}\right)-T f\right) \leq k\left(\limsup _{n \rightarrow \infty}\left(\rho^{2}\left(T f-T^{n-1}\left(f_{0}\right)\right)+\limsup _{n \rightarrow \infty} \rho^{2}\left(T^{n}\left(f_{0}\right)-f\right)\right)\right. \text {. }
$$

Thus we have

$$
\tau^{2}(T f) \leq k \tau^{2}(T f)+k \tau^{2}(f)
$$


which implies

$$
\tau^{2}(T f) \leq \frac{k}{1-k} \tau^{2}(f)
$$

Since $\frac{k}{1-k}<1$, we obtain $\tau(T f) \leq \tau(f)$. Thus, $\tau\left(T g_{n}\right) \leq \tau\left(g_{n}\right)$. Hence, $\left\{T\left(g_{n}\right)\right\}$ is also a minimizing sequence of $\tau$.

Again, according to Lemma 1.3, $\left\{g_{n}\right\}$ converges to some $g$ in $\mathbb{L}_{\rho}$ and if there is any other minimizing sequence it also converges to $g$ then $\lim _{n \rightarrow \infty} T g_{n}=g$. Next, we show that $g$ is the $\rho$-attractive element of $T$.

From Definition 2.1 and uniform continuity of $\rho$, we have

$$
\lim _{n \rightarrow \infty} \rho^{2}\left(T g_{n}-T f\right) \leq k \lim _{n \rightarrow \infty} \rho^{2}\left(T f-g_{n}\right)+k \lim _{n \rightarrow \infty} \rho^{2}\left(f-T g_{n}\right) .
$$

Therefore,

which implies

$$
\rho^{2}(g-T f) \leq k \rho^{2}(T f-g)+k \rho^{2}(f-g)
$$

$$
(1-k) \rho^{2}(g-T f) \leq k \rho^{2}(f-g)
$$

Consequently,

$$
\rho(T f-g) \leq \rho(f-g)
$$

Hence, $g$ is a $\rho$-attractive element of $T$.

As an immediate consequence of Theorem 2.1, we obtain the next result.

Theorem 2.2. Assume that $\mathbb{L}_{\rho}$ is complete, $\rho \in \Re$ is (UUC1) and uniformly continuous. Let $E$ be a nonempty $\rho$-bounded, $\rho$-closed and convex subset of $\mathbb{L}_{\rho}$. Let $T: E \rightarrow E$ be a $\rho-k$-nonspreading mapping with $k \in\left(0, \frac{1}{2}\right]$. Then $T$ has a fixed point.

Theorem 2.3. Let $\rho \in \Re$ satisfy $(U U C 2)$ and $\Delta_{2}$-condition. Let $E$ be a nonempty convex subset of $\mathbb{L}_{\rho}$ and $T: E \rightarrow \mathbb{L}_{\rho}$ be a $\rho-k$-nonspreading mapping with $k \in\left(0, \frac{1}{2}\right]$. Suppose $A_{\rho}(T)$ is nonempty and let $\left\{f_{n}\right\}$ be defined by

$$
\begin{aligned}
f_{n+1} & =\alpha_{n} T f_{n}+\left(1-\alpha_{n}\right) T g_{n}, \\
g_{n} & =\beta_{n} f_{n}+\left(1-\beta_{n}\right) T f_{n},
\end{aligned}
$$

with $0<\alpha_{n}, \beta_{n}<1$, then $\lim _{n \rightarrow \infty} \rho\left(f_{n}-h\right)$ exists for $h \in A_{\rho}(T)$ and $\lim _{n \rightarrow \infty} \rho\left(f_{n}-T f_{n}\right)=0$.

Proof. Let $h$ be a $\rho$-attractive point of $T$. Then by convexity of $\rho$ we have

$$
\begin{aligned}
\rho\left(f_{n+1}-h\right) & =\rho\left(\alpha_{n} T f_{n}+\left(1-\alpha_{n}\right) T g_{n}-h\right) \\
& \leq \rho\left(\alpha_{n}\left(T f_{n}-h\right)+\left(1-\alpha_{n}\right)\left(T g_{n}-h\right)\right) \\
& \leq \alpha_{n} \rho\left(T f_{n}-h\right)+\left(1-\alpha_{n}\right) \rho\left(T g_{n}-h\right) \\
& \leq \alpha_{n} \rho\left(f_{n}-h\right)+\left(1-\alpha_{n}\right) \rho\left(g_{n}-h\right) .
\end{aligned}
$$


Also,

$$
\begin{aligned}
\rho\left(g_{n}-h\right) & =\rho\left(\beta_{n} f_{n}+\left(1-\beta_{n}\right) T f_{n}-h\right) \\
& \leq \rho\left(\beta_{n}\left(f_{n}-h\right)+\left(1-\beta_{n}\right)\left(T f_{n}-h\right)\right) \\
& \leq \beta_{n} \rho\left(f_{n}-h\right)+\left(1-\beta_{n}\right) \rho\left(f_{n}-h\right) \\
& \leq \rho\left(f_{n}-h\right) .
\end{aligned}
$$

Thus, from (2.3) and (2.4) we have

$$
\rho\left(f_{n+1}-h\right) \leq \rho\left(f_{n}-h\right)
$$

Hence, $\left\{f_{n}\right\}$ is $\rho$-bounded and $\rho\left(f_{n}-h\right)$ is a nonincreasing sequence. Then $\lim _{n \rightarrow \infty} \rho\left(f_{n}-h\right)$ exists for each $h \in A_{\rho}(T)$.

Now we show that $\lim _{n \rightarrow \infty} \rho\left(f_{n}-T f_{n}\right)=0$. Suppose that

$$
\lim _{n \rightarrow \infty} \rho\left(f_{n}-h\right)=L \text {. }
$$

Since $h \in A_{\rho}(T)$, we have $\rho\left(T f_{n}-h\right) \leq \rho\left(f_{n}-h\right)$. Thus,

$$
\limsup _{n \rightarrow \infty} \rho\left(T f_{n}-h\right) \leq \limsup _{n \rightarrow \infty} \rho\left(f_{n}-h\right) .
$$

It follows,

$$
\limsup _{n \rightarrow \infty} \rho\left(T f_{n}-h\right) \leq L
$$

Also,

$$
\begin{aligned}
\rho\left(T g_{n}-h\right) & \leq \rho\left(g_{n}-h\right) \\
& \leq \rho\left(f_{n}-h\right)
\end{aligned}
$$

implies

$$
\rho\left(T g_{n}-h\right) \leq \limsup _{n \rightarrow \infty} \rho\left(T g_{n}-h\right) \leq L
$$

and

$$
\rho\left(g_{n}-h\right) \leq \rho\left(f_{n}-h\right)
$$

Thus

$$
\limsup _{n \rightarrow \infty} \rho\left(g_{n}-h\right) \leq L
$$

Therefore,

$$
\begin{aligned}
L & =\lim _{n \rightarrow \infty} \rho\left(f_{n+1}-h\right) \\
& =\lim _{n \rightarrow \infty} \rho\left(\alpha_{n} T f_{n}+\left(1-\alpha_{n}\right) T g_{n}-h\right) \\
& =\lim _{n \rightarrow \infty} \rho\left(\alpha_{n}\left(T f_{n}-h\right)+\left(1-\alpha_{n}\right)\left(T g_{n}-h\right)\right) .
\end{aligned}
$$

Then using (2.6), (2.7), (2.9) and Lemma 1.1 we have $\lim _{n \rightarrow \infty} \rho\left(T f_{n}-T g_{n}\right)=0$.

Fix $\epsilon>0$. Then there exists $n_{0} \in \mathbb{N}$ such that

$$
\rho\left(T f_{n}-T g_{n}\right)<\epsilon, \quad \text { for all } n \geq n_{0} .
$$


Now, using the definition of growth function,

$$
\begin{aligned}
\rho\left(\alpha_{n}\left(T f_{n}-T g_{n}\right)\right) & \leq \omega_{\rho}\left(\alpha_{n}\right) \rho\left(T f_{n}-T g_{n}\right) \\
& \leq \rho\left(T f_{n}-T g_{n}\right) \\
& <\epsilon .
\end{aligned}
$$

Therefore,

$$
\lim _{n \rightarrow \infty} \rho\left(\alpha_{n}\left(T f_{n}-T g_{n}\right)\right)=0
$$

Next,

$$
\begin{aligned}
\rho\left(f_{n+1}-h\right) & =\rho\left(\alpha_{n} T f_{n}+\left(1-\alpha_{n}\right) T g_{n}-h\right) \\
& =\rho\left(\alpha_{n}\left(T f_{n}-T g_{n}\right)+\left(T g_{n}-h\right)\right) .
\end{aligned}
$$

By using Theorem 1.1 and (2.10), we get

$$
\begin{aligned}
\liminf _{n \rightarrow \infty} \rho\left(f_{n+1}-h\right) & =\liminf _{n \rightarrow \infty} \rho\left(\alpha_{n}\left(T f_{n}-T g_{n}\right)+\left(T g_{n}-h\right)\right) \\
& =\liminf _{n \rightarrow \infty} \rho\left(T g_{n}-h\right) .
\end{aligned}
$$

Thus,

$$
\liminf _{n \rightarrow \infty} \rho\left(T g_{n}-h\right)=L
$$

Now,

$$
\begin{aligned}
& \liminf _{n \rightarrow \infty} \rho\left(T g_{n}-h\right) \leq \liminf _{n \rightarrow \infty} \rho\left(g_{n}-h\right), \\
\Rightarrow & L \leq \liminf _{n \rightarrow \infty} \rho\left(g_{n}-h\right) .
\end{aligned}
$$

Again, from (2.8) and (2.11),

$$
\lim _{n \rightarrow \infty} \rho\left(g_{n}-h\right)=L
$$

Consequently,

$$
\begin{aligned}
\lim _{n \rightarrow \infty} \rho\left(g_{n}-h\right) & =\lim _{n \rightarrow \infty} \rho\left(\beta\left(f_{n}-h\right)+(1-\beta)\left(T f_{n}-h\right)\right) \\
& =L .
\end{aligned}
$$

Hence, using (2.5), (2.6), (2.12) and Lemma 1.1 we get

$$
\lim _{n \rightarrow \infty} \rho\left(f_{n}-T f_{n}\right)=0 .
$$

Our next result discusses the $\rho$-convergence of the iterative process (2.2) to attractive elements of the mapping $T$ where $T$ satisfies condition $(I)$.

Definition 2.3. Let $E$ be a nonempty subset of $\mathbb{L}_{\rho}$. A mapping $T: E \rightarrow E$ is said to satisfy condition (I) if there exists a nondecreasing function $\ell:[0, \infty) \rightarrow[0, \infty)$ with $\ell(0)=0, \ell(r)>0$ for all $r \in(0, \infty)$ such that $\rho(f-T f) \geq \ell\left(\operatorname{dist}_{\rho}\left(f, A_{\rho}(T)\right)\right)$ where $\operatorname{dist}_{\rho}\left(f, A_{\rho}(T)\right)=\inf \left\{\rho(f-g): g \in A_{\rho}(T)\right\}$.

We give an example of a mapping that satisfies the condition $(I)$. 
Example 2.2. Let the set of real numbers $\mathbb{R}$ be the space modulared as $\rho(f)=|f|$. Let $E=\left\{f \in \mathbb{L}_{\rho}: 0<f<1\right\}$, define $T: E \rightarrow E$ as $T f=\frac{f}{2}$. Clearly, $T$ is $\rho-\frac{1}{4}$-nonspreading mapping. We know that an element $g \in \mathbb{L}_{\rho}$ is an attractive point of $T$ if $\rho(T f-g) \leq \rho(f-g)$ for all $f \in E$. Assume that $g \in A_{\rho}(T)$, then

$$
\begin{aligned}
\left|\frac{f}{2}-g\right| & \leq|f-g|, \\
\left|\frac{f}{2}-g\right|^{2} & \leq|f-g|^{2}, \\
\left|\frac{f}{2}-g\right|^{2}-|f-g|^{2} & \leq 0, \\
\left(\frac{f}{2}-g+f-g\right)\left(\frac{f}{2}-g-f+g\right) & \leq 0, \\
\left(\frac{3 f}{2}-2 g\right)\left(\frac{-f}{2}\right) & \leq 0 .
\end{aligned}
$$

Hence, we have $g \leq \frac{3 f}{4}$. Since $g$ must satisfy (2.13) for all $f$ such that $0<f<$ $1, g$ must be less or equal to 0 . Hence, $A_{\rho}(T)=(-\infty, 0]$. Define a continuous nondecreasing function $\ell:[0, \infty) \rightarrow[0, \infty)$ by $\ell(r)=\frac{r}{8}$. Then,

$$
\ell\left(d_{\rho}\left(f, A_{\rho}(T)\right)\right)=\ell\left(d_{\rho}(f,(-\infty, 0])\right)=\ell(|f|)=\frac{|f|}{8}<\left|\frac{f}{2}-f\right| .
$$

Hence, $\rho(f-T f) \geq \ell\left(d_{\rho}\left(f, A_{\rho}(T)\right)\right)$ for all $f \in E$.

Theorem 2.4. Let $\rho \in \Re$ satisfies (UUC2) and $\Delta_{2}$-condition. In addition, $\rho$ is uniformly continuous. Let $E$ be a nonempty convex subset of $\mathbb{L}_{\rho}$ and $T: E \rightarrow E$ be a $\rho-k$-nonspreading mapping with $k \in\left(0, \frac{1}{2}\right]$. Assume $A_{\rho}(T) \neq \phi$ and $T$ satisfies condition $(I)$. Let $\left\{f_{n}\right\}$ be defined as in (2.2), with $0<\alpha_{n}, \beta_{n}<1$. Then $\left\{f_{n}\right\}$ $\rho$-converges to a $\rho$-attractive point of $T$.

Proof. We already know $\rho\left(f_{n+1}-h\right) \leq \rho\left(f_{n}-h\right)$ and $\lim _{n \rightarrow \infty} \rho\left(f_{n}-T f_{n}\right)=0$. Then by condition $(I)$ and Theorem (2.3), we have

$$
\begin{aligned}
\liminf _{n \rightarrow \infty} \rho\left(f_{n}-T f_{n}\right) & \geq \liminf _{n \rightarrow \infty} \ell\left(d_{\rho}\left(f_{n}, A_{\rho}(T)\right),\right. \\
0 & \geq \liminf _{n \rightarrow \infty} \ell\left(d_{\rho}\left(f_{n}, A_{\rho}(T)\right) .\right.
\end{aligned}
$$

This implies $\lim _{n \rightarrow \infty} \ell\left(d_{\rho}\left(f_{n}, A_{\rho}(T)\right)=0\right.$. It follows $\lim _{n \rightarrow \infty} d_{\rho}\left(f_{n}, A_{\rho}(T)\right)=0$, since $\ell(0)=0$.

Now, we show that $\left\{f_{n}\right\}$ is $\rho$-Cauchy. Since $\lim _{n \rightarrow \infty} d_{\rho}\left(f_{n}, A_{\rho}(T)\right)=0$, let $\epsilon>0$, then there exists a constant $n_{0}$ such that for $n \geq n_{0}$

$$
d_{\rho}\left(f_{n}, A_{\rho}(T)\right)<\frac{\epsilon}{2}
$$




$$
\left\{\inf \rho\left(f_{n}-h\right): h \in A_{\rho}(T)\right\}<\frac{\epsilon}{2} .
$$

Then there must exist some $h^{*} \in A_{\rho}(T)$ such that $\rho\left(f_{n_{0}}-h^{*}\right)<\epsilon$. Now for $m, n \geq n_{0}$, we have by convexity of $\rho$ and the fact that $\rho\left(\left\{f_{n}-h\right\}\right)$ is non increasing,

$$
\begin{aligned}
\rho\left(\frac{f_{n+m}-f_{n}}{2}\right) & \leq \rho\left(\frac{\left(f_{n+m}-h\right)-\left(f_{n}-h\right)}{2}\right) \\
& \leq \frac{1}{2}\left(\rho\left(f_{n+m}-h\right)\right)+\frac{1}{2}\left(\rho\left(f_{n}-h\right)\right) \\
& <\frac{1}{2}\left(\rho\left(f_{n_{0}}-h^{*}\right)\right)+\frac{1}{2}\left(\rho\left(f_{n_{0}}-h^{*}\right)\right) \\
& =\rho\left(f_{n_{0}}-h^{*}\right) \\
& <\epsilon .
\end{aligned}
$$

Hence, by $\Delta_{2}$-condition, $\left\{f_{n}\right\}$ is a $\rho$-Cauchy sequence. Since $\mathbb{L}_{\rho}$ is complete, the sequence $\left\{f_{n}\right\} \rho$-converges to some $q$ in $\mathbb{L}_{\rho}$.

Let $\lim _{n \rightarrow \infty} \rho\left(f_{n}-q\right)=0$. Then, by convexity of $\rho$ and Theorem 2.3,

$$
\lim _{n \rightarrow \infty} \rho\left(T f_{n}-q\right)=0 .
$$

Further, by definition (2.1) and uniform convexity of $\rho$, we get the following

$$
\lim _{n \rightarrow \infty} \rho^{2}\left(T f_{n}-T f\right) \leq k \lim _{n \rightarrow \infty} \rho^{2}\left(f_{n}-T f\right)+k \lim _{n \rightarrow \infty} \rho^{2}\left(T f_{n}-f\right) .
$$

This implies

$$
\rho^{2}(q-T f) \leq k \rho^{2}(q-T f)+k \rho^{2}(q-f)
$$

This results

$$
\rho(q-T f) \leq \frac{k}{1-k} \rho(q-f) \leq \rho(q-f) .
$$

Hence, $q \in A_{\rho}(T)$ and $\lim _{n \rightarrow \infty} \rho\left(f_{n}-q\right)=0$.

Let $E$ be a subset of $\mathbb{L}_{\rho}$. A mapping $T: E \rightarrow \mathbb{L}_{\rho}$ is said to be $\rho$-demicompact if it has the property that whenever a sequence $\left\{f_{n}\right\} \in E$ is $\rho$-bounded and the sequence $\left\{f_{n}-T f_{n}\right\} \rho$-converges, then there exists a subsequence $\left\{f_{n_{k}}\right\}$ which is $\rho$-convergent.

Theorem 2.5. Let $\rho \in \Re$ satisfies (UUC2) and $\Delta_{2}$-condition. In addition, let $\rho$ is uniformly continuous. Let $E$ be a nonempty convex subset of $\mathbb{L}_{\rho}$ and $T: E \rightarrow E$ be a $\rho$-k-nonspreading with $k \in\left(0, \frac{1}{2}\right]$ and $\rho$-demicompact mapping with $A_{\rho}(T) \neq \phi$. Let $\left\{f_{n}\right\}$ be defined as in (2.2) with $0<\alpha_{n}, \beta_{n}<1$. Then $\left\{f_{n}\right\} \rho$-converges to a $\rho$-attractive point of $T$.

Proof. From Theorem 2.3 we already know that $\left\{f_{n}\right\}$ is a bounded sequence and $\lim _{n \rightarrow \infty} \rho\left(f_{n}-T f_{n}\right)=0$. Then by demicompactness of operator $T$ there exists a subsequence $\left\{f_{n_{k}}\right\}$ of $\left\{f_{n}\right\}$ and $g \in \mathbb{L}_{\rho}$ such that $\lim _{n \rightarrow \infty} \rho\left(f_{n_{k}}-g\right)=0$. Also, by uniform continuity of $\rho$ and since $\lim _{n \rightarrow \infty} \rho\left(f_{n}-T f_{n}\right)=0$, we have

$$
\lim _{n \rightarrow \infty} \rho\left(T f_{n_{k}}-g\right)=0 \text {. }
$$


Now, by definition of $\rho-k$-nonspreading mapping and uniform continuity of $\rho$ we have,

$$
\lim _{n \rightarrow \infty} \rho^{2}\left(T f_{n_{k}}-T f\right) \leq k \lim _{n \rightarrow \infty} \rho^{2}\left(f_{n_{k}}-T f\right)+k \lim _{n \rightarrow \infty} \rho^{2}\left(T f_{n_{k}}-f\right) .
$$

Consequently,

$$
\rho^{2}(g-T f) \leq k \rho^{2}(g-T f)+k \rho^{2}(g-f) .
$$

That is,

$$
\rho(g-T f) \leq \rho(g-f)
$$

So, $g \in A_{\rho}(T)$. By Theorem 2.3, if $\lim _{n \rightarrow \infty} \rho\left(f_{n}-g\right)$ exists for any $g \in A_{\rho}(T)$, then we have, $\lim _{n \rightarrow \infty} \rho\left(f_{n}-g\right)=0$.

\section{Numerical Results}

Now the following examples verify the results in Theorems 2.4 and 2.5.

Example 3.1. Let the set of real numbers $\mathbb{R}$ be the space modulared as $\rho(f)=|f|$. Let $E=\left\{f \in \mathbb{L}_{\rho}: 0<f<1\right\}$, define $T: E \rightarrow E$ as $T f=\frac{f}{2}$. Obviously, $E$ is a nonempty convex subset of $\mathbb{R}$ which satisfies $(U C 1)$ condition. Also $\rho(f)=|f|$ is uniformly continuous and ( $U U C 2)$ holds. We have already seen $A_{\rho}(T)$ is nonempty. Finally, we generate the sequence (2.2) and show that it converges to its attractive point. Choose $f_{1}=0.3125$ and $\alpha=\beta=\frac{1}{2}$, then we have the results in Table 1 .

TABLE 1. Numerical results of Example 3.1

\begin{tabular}{|c|c|}
\hline$n$ & $f_{n}$ \\
\hline 1 & 0.312500000000000 \\
2 & 0.136718750000000 \\
3 & 0.059814453125000 \\
4 & 0.026168823242188 \\
5 & 0.011448860168457 \\
6 & 0.005008876323700 \\
7 & 0.002191383391619 \\
$\vdots$ & $\vdots$ \\
48 & $4.176559929877658 \mathrm{e}-18$ \\
49 & $1.827244969321475 \mathrm{e}-18$ \\
50 & $7.994196740781455 \mathrm{e}-19$ \\
\hline
\end{tabular}

This shows that $\left\{f_{n}\right\}$ converges to $0 \in A_{\rho}(T)$. This is worth mentioning here that $T$ does not have any fixed point in $D$. 
Example 3.2. Let the set of real numbers $\mathbb{R}$ be the space modulared as $\rho(f)=|f|^{k}$. Let $E=\left\{f \in \mathbb{L}_{\rho}:-3<f<2\right\}$, define $T: E \rightarrow E$ as:

$$
T f= \begin{cases}\frac{|f|-1}{2}, & -2<f<2, \\ \frac{-|f|}{|f|+1}, & -3<f \leq-2 .\end{cases}
$$

Obviously, $E$ is a nonempty convex subset of $\mathbb{R}$ which satisfies (UC1) condition. Also $\rho(f)=|f|^{k}$ is uniformly continuous and (UUC2) holds. Since the mapping is $\rho$-quasi nonexpansive and $F(T)=\left\{-\frac{1}{3}\right\}$ then $A_{\rho}(T) \neq \phi$. $T$ is $\rho$-demicompact since any sequence $\left\{f_{n}\right\} \in(-3,-2)$ is bounded, i.e., $\left|f_{n}\right|<3$ and any bounded sequence in $\mathbb{R}$ has a convergent subsequence. Now finally, we generate the sequence (2.2) and show that it converges to its attractive point. Choose $f_{1}=1.5$ and $\alpha=\beta=\frac{1}{2}$, then we have the results in Table 2. This shows that $\left\{f_{n}\right\}$ converges to $-\frac{1}{3} \in A_{\rho}(T)$.

TABLE 2. Numerical results of Example 3.2

\begin{tabular}{|c|c|}
\hline$n$ & $f_{n}$ \\
\hline 1 & 1.5 \\
2 & 0.093750000000000 \\
3 & -0.431640625000000 \\
4 & -0.302612304687500 \\
5 & -0.342933654785156 \\
6 & -0.330333232879639 \\
7 & -0.334270864725113 \\
$\vdots$ & $\vdots$ \\
28 & -0.333333333333310 \\
29 & -0.333333333333341 \\
30 & -0.333333333333331 \\
\hline
\end{tabular}

\section{REFERENCES}

[1] W. I. A. Kaewkhao and K. Kunwai, Attractive points and convergence theorems for normally generalized hybrid mappings in cat (0) spaces, Fixed Point Theory Appl. 2015(1) (2015), 14 pages.

[2] B. A. B. Dehaish and W. M. Kozlowski, Fixed point iteration processes for asymptotic pointwise nonexpansive mapping in modular function spaces, Fixed Point Theory Appl. 2012(118) (2012), 23 pages.

[3] A. Ilchev and B. Zlatanov, Fixed and best proximity points for kannan cyclic contractions in modular function spaces, J. Fixed Point Theory Appl. 9 (2017), 2873-2893.

[4] A. Ilchev and B. Zlatanov, Coupled fixed points and coupled best proximity points in modular function spaces, International Journal of Pure and Applied Mathematics 118 (2018), 957-977.

[5] A. K. K. Kunwai and W. Inthakon, Properties of attractive points in cat (0) spaces, Thai J. Math. 13 (2015), 109-121. 
[6] M. A. Khamsi, Convexity property in modular function spaces, Sci. Math. Jpn. 44 (1996), 269-280.

[7] M. A. Khamsi and W. M. Kozolowski, Fixed Point Theory in Modular Function Spaces, Springer, Berlin, 2015.

[8] F. Kohsaka and W. Takahashi, Fixed point theorems for a class of nonlinear mappings related to maximal monotone operators in banach spaces, Arch. Math. 91 (2008), 166-177.

[9] W. M. Kozlowski, Advancements in fixed point theory in modular function spaces, Arab. J. Math. 1 (2012), 477-494.

[10] K. Kuaket and P. Kumam, Fixed points of asymptotic pointwise contractions in modular spaces, Appl. Math. Lett. 24 (2011), 1795-1798.

[11] M. A. Khamsi, W. M. Kozlowski and S. Reich, Fixed point theory in modular function spaces, Nonlinear Anal. 14 (1990), 935-953.

[12] J. Musielak and W. Orlicz, On modular spaces, Studia Math. 18 (1959), 49-65.

[13] H. Nakano, Modular Semi-Ordered Spaces, Maruzen, Tokyo, 1950.

[14] S. H. Khan, M. Abbas and S. Ali, Fixed point approximation of multivalued $\rho$-quasi-nonexpansive mappings in modular function spaces, J. Nonlinear Sci. Appl. 10 (2017), 3168-3179.

[15] S. Suantai, P. Cholamjiak, Y. J. Cho and W. Cholamjiak, On solving split equilibrium problems and fixed point problems of nonspreading multi-valued mappings in hilbert spaces, Fixed Point Theory Appl. 2016 (2016), 35.

[16] W. Takahashi and Y. Takeuchi, Nonlinear ergodic theorem without convexity for generalized hybrid mappings in a hilbert space, J. Nonlinear Convex Anal. 12 (2011), 399-406.

[17] W. Takahashi, N. C. Wong and J. C. Yao, Attractive point and weak convergence theorems for new generalized hybrid mappings in Hilbert spaces, J. Nonlinear Convex Anal. 13 (2012), $745-757$.

[18] Y. Zheng, Attractive points and convergence theorems of generalized hybrid mapping, J. Nonlinear Sci. Appl. 8 (2015), 354-362.

[19] B. Zlatanov, Best proximity points in modular function spaces, Arab. J. Math. 4 (2015), 215-227.

${ }^{1}$ Department of Sciences and Humanities,

National University of Computer and Emerging Sciences, Lahore Campus,

Block B, Faisal Town, Lahore, Pakistan

Email address: hira.iqbal@nu.edu.pk

${ }^{2}$ Department of Mathematics,

Government College University Lahore,

54000 LAHORE, PAKISTAN

${ }^{2}$ Department of Mathematics,

King Abdulaziz University,

P. O. Box. 80203, Jeddah 21589, SAudi Arabia

Email address: abbas.mujahid@gmail.com

${ }^{3}$ Department of Mathematics, Statistics and Physics,

QATAR UNIVERSITY,

Doha, 2713, SATE of QATAR

Email address: safeer@qu.edu.qa 